\title{
Simulation in Surgical Education
}

\author{
Sandra L. de Montbrun, M.D. ${ }^{1}$ Helen MacRae, M.D. ${ }^{2}$ \\ 1 St. Michael's Hospital, Toronto, Ontario, Canada \\ 2 Mount Sinai Hospital, Joseph and Wolf Lebovic Health Complex, \\ Toronto, Ontario, Canada \\ Clin Colon Rectal Surg 2012;25:156-165.

\begin{abstract}
Address for correspondence and reprint requests Sandra L. de Montbrun, M.D., St. Michael's Hospital, 30 Bond St., Rm. 16-064, Cardinal Carter Wing, Toronto, Ontario, M5B 1W8, Canada (e-mail: demontbrunsa@smh.ca).
\end{abstract}
Abstract
Keywords
- simulation
- surgical education
- bench models
- virtual reality
- nontechnical skills

The pedagogical approach to surgical training has changed significantly over the past few decades. No longer are surgical skills solely acquired through a traditional apprenticeship model of training. The acquisition of many technical and nontechnical skills is moving from the operating room to the surgical skills laboratory through the use of simulation. Many platforms exist for the learning and assessment of surgical skills. In this article, the authors provide a broad overview of some of the currently available surgical simulation modalities including bench-top models, laparoscopic simulators, simulation for new surgical technologies, and simulation for nontechnical surgical skills.
Objectives: Upon completion of this article, the reader should have an understanding of the definition and application of simulation as it relates to surgical training and be able to summarize the simulation platforms that are currently available for both technical and nontechnical surgical skills.

Achieving surgical competence is a complex process that involves the attainment of knowledge, judgment, professionalism, and surgical skill. ${ }^{1}$ For the past century, surgeons have been taught through the Halstedian model of surgical training, which involved learning the craft of surgery through apprenticeship. ${ }^{2,3}$ Although this model of surgical education has been successful at providing a skilled surgical workforce in the past, a multitude of factors have influenced the need to reconsider pedagogical strategies in surgical education. Surgical training is undergoing a paradigm shift, with the movement of the acquisition phase of many basic surgical skills from the operating room (OR) to the surgical skills laboratory. As well, nontechnical skills, such as team communication, crisis resource management, and interpersonal communication skills are increasingly being addressed using simulation. ${ }^{4}$ In this article, we will provide a broad overview of surgical simulation, including bench-top models, laparoscopic simulators, simulation for new surgical technologies, and simulation for nontechnical skills.

\section{What Is Simulation?}

Simulation is an educational technique that allows the interactive performance of the trainee in an environment that recreates or replicates a real-world clinical scenario, but is not identical to "real life." ${ }^{\text {,6 }}$ Simulation can include anything from the use of standardized patients and low-fidelity bench models, to high-fidelity technologically advanced virtual reality (VR) systems. Simulation has been used and is currently well established in several other industries including the military, aviation, and astronautics. ${ }^{5}$ The use of simulation in these industries predates its use in surgery with simulation in aviation dating back to the 1950s. Pilots must undergo exhaustive drills and certification on flight simulators before being allowed to fly a plane. For example, the first Boeing 777 flight was flown by a pilot with over 1000 hours of simulation training. ${ }^{7}$ Although lagging behind other industries, the use of simulation in surgical training has recently gained significant momentum.

\section{The Need for Simulation in Surgery}

Perhaps one of the most compelling driving forces for the integration of simulation into surgical training is the ethical imperative of providing patients with the best care. Although it is understood that trainees will eventually develop technical skills by treating patients, patients should not be subjected to the possibility of harm when other training methods are available for skill acquisition. ${ }^{8}$ Simulation ensures that some practice has taken place before trainees treat real patients. ${ }^{5}$ Simulation also allows for alternate ways to acquire skills within the constraints of work-hour restrictions and limited clinical exposure. Although data suggests that surgical
Issue Theme Surgical Education in Colorectal Surgery; Guest Editor, Judith L. Trudel, M.D.
Copyright (c) 2012 by Thieme Medical Publishers, Inc., 333 Seventh Avenue, New York, NY 10001, USA. Tel: +1(212) 584-4662.
DOI http://dx.doi.org/ 10.1055/s-0032-1322553. ISSN 1531-0043. 
residents' operative volume in the United States has not decreased, ${ }^{9}$ it has been argued that the current case volume may not be adequate for surgical training, especially when new technologies are introduced. ${ }^{10,11}$ Simulators are available at any time to be used, making them flexible for training, unlike patient exposure. ${ }^{5}$ Furthermore, operating room teaching is both time consuming and expensive. A study by Bridges et al estimated that the increase in operative time attributed to resident training translated into \$53 million dollars a year. ${ }^{12}$ Simulation can also provide a means for both residents and surgeons in practice to acquire the necessary skills to incorporate new surgical technologies and innovation into their surgical repertoire, with the resultant shift of the learning curve away from the patient. Simulation allows room for error and the ability to allow trainees to learn the consequences of error because incorrect tasks can be performed to completion. ${ }^{13}$ Finally, important skills such as crisis resource management and team training can be practiced away from the emergency setting, allowing for better team functioning when crises occur. Various types of simulations are available, including bench-top models, simulators to teach laparoscopic skills and to teach new technologies, and simulations for nonsurgical skills and team training.

\section{Bench-Top Models}

Bench-top models are designed to teach surgical procedures or assess technical performance, using synthetic material or nonlive animal tissue. One of the earliest reported bench-top models was described in 1986 by Pikal who demonstrated the ability to improve surgical performance using freeze-dried porcine tissue to teach intestinal anastomosis. ${ }^{14}$ In the mid1990s, Reznick et al at the University of Toronto ${ }^{15}$ developed an objective assessment of technical skill (OSATS) using benchtop models of either synthetic material or nonlive animal tissue. Models were developed to assess a variety of technical procedures including excision of a skin lesion, bowel anastomosis, insertion of a $\mathrm{T}$ tube, and abdominal wall closure (-Fig. 1). The OSATS has demonstrated the validity and reliability of bench-top models in the assessment of technical skills. ${ }^{16-19}$ OSATS models were initially developed for general surgery; however, they have now been developed for other surgical specialties. ${ }^{20}$ Obstetrics and gynecology have also developed and evaluated the psychometric properties of inexpensive bench-top models. ${ }^{21}$ Advanced hysteroscopic surgical skills of endometrial resection and ablation have been practiced on a low-fidelity, inexpensive butternut pumpkin mod$\mathrm{el}^{22}$ and the surgical repair of obstetrical anal sphincter injury has been simulated using porcine tongue. ${ }^{23}$

Although high-fidelity laparoscopic VR models are alluring, bench-top models continue to be developed for many training needs. In a recent randomized control trial, Lauscher et al described the use of a bench-top simulator-the Berlin Operation Trainer (BOPT) for training conventional open gastrointestinal techniques including bowel anastomosis. The BOPT mimics the reality of the OR requiring the trainee to operate standing up within the confines of simulated anatomy such as the pelvis and upper abdomen. ${ }^{24}$ Compared

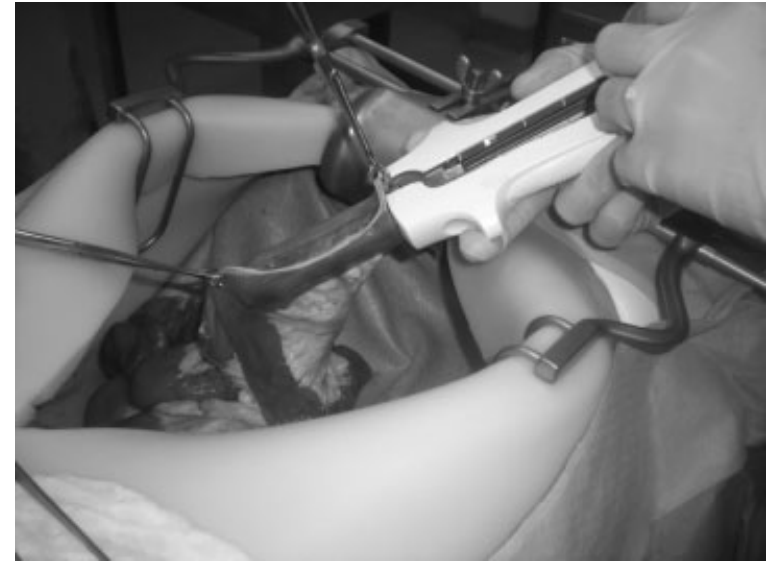

Figure 1 Bench-top model demonstrating a side-to-side stapled anastomosis using porcine small bowel.

with the control group who were trained on conventional models, the BOPT-trained group demonstrated a significant improvement in terms of speed and overall performance score. Interestingly, even those participants who were classified as experts demonstrated improved performance after training on the BOPT, suggesting that bench-top models can impact skill acquisition at higher levels of training. Evidence of potential transfer of skills to the OR was suggested by demonstrating improved performance in an anesthetized pig. This same group has gone on to further establish face, content, and construct validity of the BOPT. ${ }^{25}$

There is considerable evidence that bench- top training leads to skill acquisition. A prospective cohort study by Jensen et al demonstrated that simulation-based training for excision of a skin lesion and small bowel anastomosis on a porcine model resulted in a significant improvement in performance with a decreased time to completion, improved overall global score, and for the anastomosis, an increase in anastomotic leak pressure. ${ }^{26}$ Bench-top model training has also been shown to significantly improve ureteroscopic techniques. ${ }^{27}$ A randomized, single-blinded trial by Anastakis et al demonstrated that bench-top models can improve performance among first-year surgical residents in a variety of open surgical procedures including small bowel anastomosis, abdominal wall closure, and flexor tendon repair. ${ }^{28}$ Grober at al studied bench-top model training in urological microsurgery using both a high-fidelity live rat vas deferens and a low-fidelity silicone tubing model, demonstrating significant improvement in skill over traditional didactic teaching, ${ }^{29}$ which persisted at 4-month follow-up. ${ }^{30}$ For the most part, bench-top models have focused on and been shown to improve technical skills in junior trainees; however, some models have shown to improve skills in more senior trainees as well ${ }^{24}$ suggesting the utility of bench-top models across different levels of training.

The importance of fidelity of bench-top models or level of "realism" in terms of visual representation and tissue texture has also been addressed. ${ }^{31}$ Low-fidelity models bear little resemblance to actual human anatomy, whereas high-fidelity 
models aim to replicate human anatomy and tissue as closely as possible. Regardless of model fidelity, it is crucial that bench models replicate the critical steps of a given procedure. Skill acquisition, for the most part, has been shown to be equivalent for both low-fidelity and high-fidelity bench-top models, with both levels of fidelity outperforming traditional didactic teaching. ${ }^{28,29,32}$ However, a randomized controlled trial by Sidhu et al demonstrated that model fidelity did have an impact on skill acquisition, with those who trained on a high-fidelity vascular anastomosis model outperforming the low-fidelity trained group. ${ }^{33}$ This suggests that the importance of model fidelity may be procedure specific. Further study is needed to help understand how fidelity impacts skill acquisition.

The ultimate goal of surgical simulation is not simply the acquisition of skill, but how well the skill training transfers to the live operation. Several studies have used performance on live anesthetized animals or cadaveric models as surrogates for operative performance. ${ }^{24,28}$ Taking transfer one step further, Datta et al compared saphenofemoral dissection performance on a bench-top synthetic model to live performance in the OR. They found that technical skill on the laboratory model correlated very closely with live operative performance, providing evidence that bench-top models validly assess surgical technical ability. ${ }^{34}$ Also, Palter et al showed that learning abdominal fascial closure on a lowfidelity synthetic bench-top model translated into improved technical performance in the OR. ${ }^{35}$

There are several advantages to using bench-top models in surgical education. They allow residents to achieve proficiency through unlimited practice opportunities. As well, they allow for improvement in training efficiency in the operating room by learning some skills outside of the OR. For example, a randomized controlled trial by Palter et al demonstrated that training to proficiency on a low-fidelity bench-top model allowed trainees to focus on other aspects of learning while in the OR. ${ }^{35}$ Bench-top models also have the advantage that they can often be made from inexpensive synthetic materials and thus are more affordable in most cases than VR models. Another benefit is that residents gain familiarity with the same type of equipment they will be using in the OR. From a practical standpoint, most open procedures do not have a VR simulation available, and cadaver or live animal models are expensive and not always available.

From a global perspective, bench-top models have significant appeal. Because many bench-top models can be made inexpensively from synthetic materials, this modality of teaching technical skills is more feasible for augmenting training in developing countries with limited resources. Dorman et al demonstrated that low-fidelity bench-top simulation training aided the training of general surgery residents in Ethiopia with a feasible, cost-effective, and safe program. ${ }^{36}$

\section{Simulation for Laparoscopic Surgery}

Much of the push for simulation in laparoscopic surgery developed after the mass introduction of laparoscopic chole- cystectomy in the late 1980s and early 1990s. Many surgeons were taking on this new skill without previous experience, resulting in an increased risk in bile duct injury during the early learning curve, with $90 \%$ of injuries occurring during the first 30 cases. ${ }^{37}$ There are several technical challenges that are faced when performing laparoscopic surgery. The surgeon must operate with little tactile feedback, from a two-dimensional image, with long instruments that move paradoxically with hand movement. Simulation has offered the opportunity to acquire laparoscopic skills in a safe environment outside of the OR, placing surgeons farther along their learning curve before operating on patients. ${ }^{38}$

Several simulation modalities are available for the acquisition of laparoscopic skills. These vary from partial task trainers that allow for the development of skills that are common to many laparoscopic procedures (knot tying, suturing, clipping) to procedure-specific trainers that are intended to teach entire operations such as a laparoscopic appendectomy, cholecystectomy, and hysterectomy. They also vary in their level of fidelity from inexpensive low-fidelity box trainers to high-fidelity VR simulators.

\section{Low-Fidelity Laparoscopic Trainers}

Gaining basic laparoscopic skills such as transferring an object, cutting, and clipping are essential tasks and can be achieved with the use of a very basic low-fidelity laparoscopic setup. Basic training sets consist of a training box, laparoscopic instruments, a camera, and light source. Khine et al have demonstrated that a portable basic training box can be constructed easily and inexpensively with widely available material using a high-definition webcam, plastic storage box, and fluorescent light source. ${ }^{39}$ Furthermore, Kobayashi et al have demonstrated construct validity and skill acquisition with this type of portable, personal laparoscopic trainer. ${ }^{40}$ With international work-week restrictions, the concept of an affordable, portable "homemade" laparoscopic skills training box is appealing, making the acquisition of laparoscopic skills more accessible for all trainees.

Several formal laparoscopic skills training models have been developed. Perhaps one of the most established and validated is the McGill Inanimate System for Training and Evaluation of Laparoscopic Skills (MISTELS). Developed in 1998 by Fried's group, this basic laparoscopic trainer simulates technical skills that are essential to the practice of laparoscopic surgery and is made up of a box-trainer, which uses laparoscopic instruments and a laparoscopic camera. ${ }^{41}$ Tasks include peg transfer, pattern cutting, clipping and dividing, placement of a ligating loop, placing and fixing a mesh, and suturing with intra- and extracorporeal knots (-Fig. 2). Performance is graded based on both precision and speed. The initial study of the MISTELS demonstrated evidence of construct validity with more experienced surgeons performing significantly better than their junior counterparts. ${ }^{41}$ Evidence of construct validity has also been demonstrated in specialties other than general surgery such as urology and gynecology, ${ }^{42,43}$ allowing the MISTELS to be used in a variety of surgical training programs. 


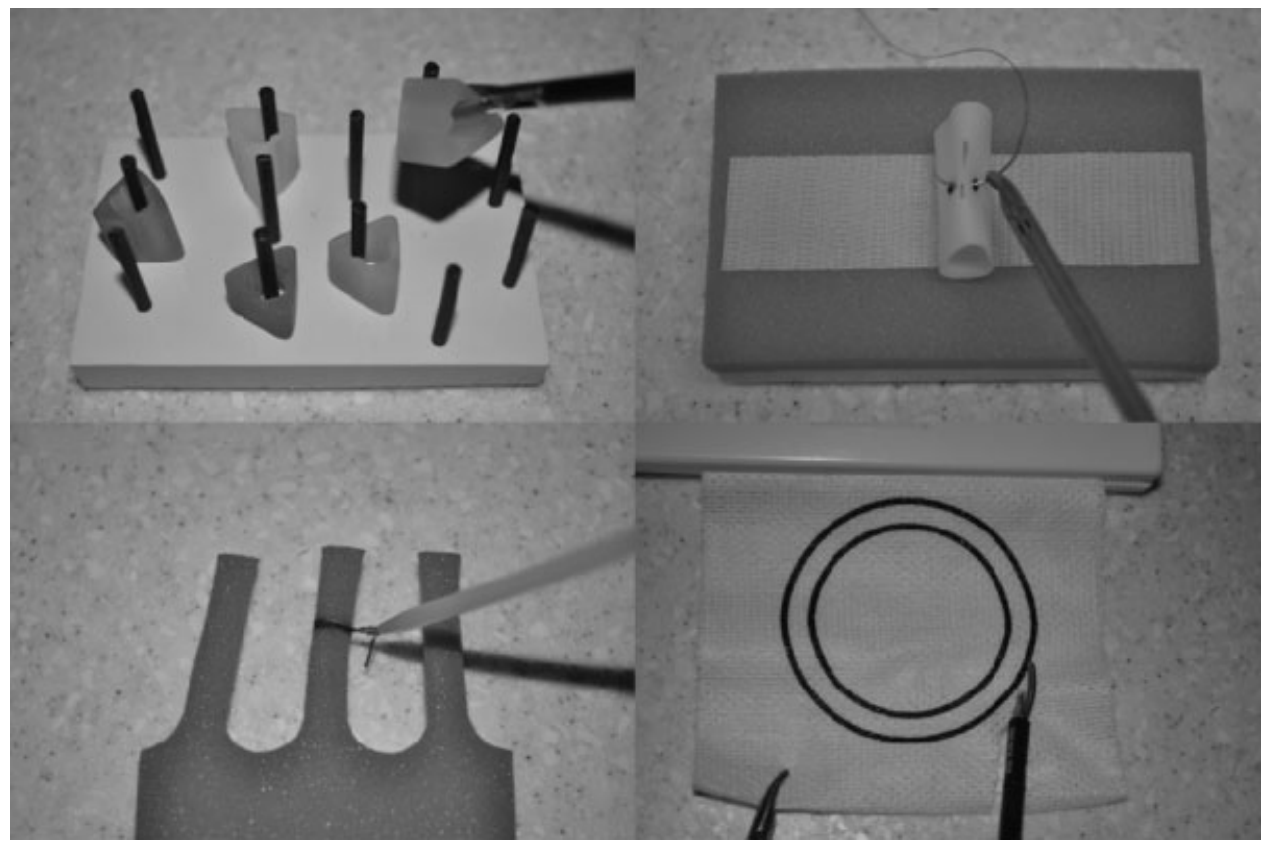

Figure 2 Basic laparoscopic skills tasks including peg transfer, intracorporeal suturing, placement of a ligating loop, and pattern cutting.

The value of laparoscopic box-training using the MISTELS system has been established. Evidence of skill acquisition has been demonstrated, showing that practice using the MISTELS platform improves performance, ${ }^{44}$ and that MISTELS scores correlate with performance on both animal models ${ }^{45}$ and live OR performance. ${ }^{46}$ Furthermore, a recent randomized controlled trial by Sroka et al demonstrated that skills acquired from MISTELS training transfer to OR performance. ${ }^{47}$ General surgery residents who were trained using the MISTELS system had a statistically significant improvement over the untrained group in OR performance, which was assessed during the dissection of the gallbladder off the liver bed during an elective laparoscopic cholecystectomy.

The MISTELS metrics have been extensively investigated and have proven to be highly reliable and valid, in the range acceptable for high stakes examinations. ${ }^{48}$ Furthermore, pass/ fail rates have been established. ${ }^{49}$ The robust psychometric properties and the established passing score of the MISTELS have allowed it to become the basis for the technical skills component of the Fundamentals of Laparoscopic Surgery (FLS) program. One of the greatest advantages of the MISTELS system is its technical simplicity, affordability, and portability.

\section{Virtual Reality Laparoscopic Trainers}

Virtual reality trainers for laparoscopic surgery exist as partial task trainers that emphasize psychomotor skill acquisition (MIST-VR) or as both partial task and full-procedure trainers (LapSim, LAP Mentor) (-Fig. 3). Most VR systems compute a score using time to completion and error measures as indicators of performance.

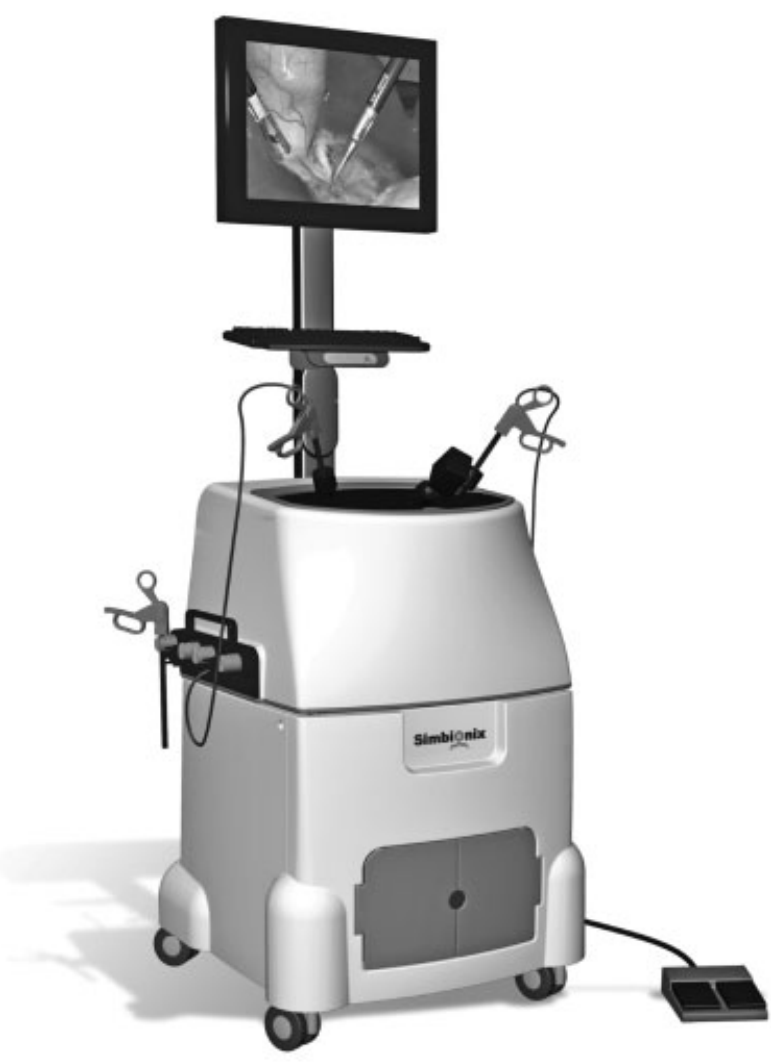

Figure 3 LAP Mentor ${ }^{\mathrm{TM}}$ virtual reality platform. Image reproduced with permission from Simbionix Inc. (Cleveland, $\mathrm{OH}$ ). 


\section{Partial Task Training}

Construct validity for most VR simulators has been demonstrated with experienced surgeons outperforming trainees and nonsurgeons. Expert surgeons make fewer correctional movements, are more efficient, and complete tasks fasters than nonexperts. ${ }^{50-54}$ Performance parameters measured with VR training have also been shown to strongly correlate with OR performance. ${ }^{55,56}$ Furthermore, trials have demonstrated decreased error and improved efficiency of trained residents compared with nontrained control groups..$^{50,57}$ Several randomized controlled trials have investigated whether skills learned on a VR trainer transfer to improved operative performance. Seymour et al demonstrated that surgical trainees randomized to MIST-VR training were 29\% faster at dissecting the gallbladder from the liver bed $(p>0.05)$. Furthermore, the nontrained group was significantly more likely to fail to progress, injure the gallbladder, and burn nontarget tissue. ${ }^{58}$ Grantcharov et al also demonstrated that skills transfer to the OR, showing that trainees who were randomized to MIST-VR training subsequently outperformed their nontrained counterparts during the dissection of gallbladder off the liver bed. The VR-trained group was significantly faster than the control group and demonstrated a significantly greater improvement in OR performance in terms of economy of motion and error. ${ }^{59}$ Andreatta et al in a randomized controlled trial allocated surgical interns to VR training in basic skills on the LAP Mentor or to no VR training. Performance was measured on a live porcine model (as a surrogate for OR performance) and demonstrated that the VR-trained group performed significantly better than the untrained group in a variety of assessed measures including accuracy and time. ${ }^{60}$

\section{Procedure Training}

One of the first procedure-specific simulators was a VR platform introduced by Delp et al and dates back to 1990. A graphical interface allowed for the practice of simulated tendon transfers and lengthening procedures to be performed on a graphic-based lower extremity. ${ }^{61}$ Since that time, significant advancements have been made in VR training. Some of the full-procedure models currently available include laparoscopic cholecystectomy, appendectomy, sigmoidectomy, ventral hernia, gastric bypass, and several gynecological procedures ( - Fig. 4).

Most studies validating VR training have focused on basic skills training rather than procedural training. One randomized controlled trial by Larsen et al trained the intervention group using a full-procedure VR right-sided salpingectomy. Performance of both the intervention group and control group was then assessed during the candidates' first live salpingectomy. They found that the simulator-trained group performed significantly better and in half the time compared with the untrained group $(p<0.001)$ demonstrating the benefit of procedural VR training. ${ }^{62}$

\section{Comparing Low-Fidelity Training to VR Training}

A recent nonrandomized prospective trial by Beyer et al compared LAP Mentor training, MISTELS training, and conven-

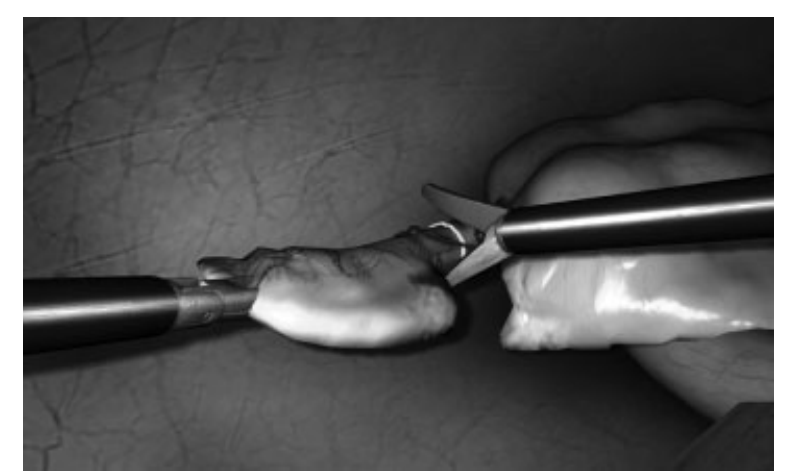

Figure 4 A screenshot from the laparoscopic appendectomy module from the LapSim ${ }^{\circledR}$ virtual reality platform. Image reproduced with permission from Surgical Science Inc. (Minneapolis, MN).

tional training with the goal of assessing the impact of simulation on operative performance as measured during a live laparoscopic cholecystectomy. ${ }^{63}$ They demonstrated that the LAP Mentor and MISTELS groups performed significantly better than the control group suggesting that both modes of simulator training resulted in improved operative performance with no significant difference between LAP Mentor or MISTELS training.

Several systematic reviews have recently been published looking at the effectiveness of surgical simulation in surgical training with varying results. A systematic review by Sutherland et al evaluated the effectiveness of various types of surgical simulation and found that VR simulation training resulted in better performance than no training; however, VR training was not found to be consistently better than either standard training (didactic teaching/training videos/surgical drills) or laparoscopic box-training. ${ }^{64}$ In contrast, a recent Cochrane systematic review of VR training in surgical education found that VR training, in comparison to standard training, decreased operative time, increased accuracy, and economy of movement in individuals with limited laparoscopic training. In parallel with Sutherland et al, they also found that VR training was at least as effective as laparoscopic boxtraining, and suggested VR training as a way to supplement current training methods. ${ }^{65}$

Although further evidence is needed to elucidate a clear benefit of expensive high-fidelity VR trainers over inexpensive, low-fidelity, box-trainers such as the MISTELS, several advantages do exist. VR simulators eliminate the cost and time commitment of human preceptors that are required to assess performance using box-trainers by producing computer-generated objective performance metrics. ${ }^{66}$ They can track a trainee's performance over time and compare their performance with that of others. However, the metrics provided can be difficult for the learner to interpret and thus limit the usefulness of its formative feedback. Furthermore, the cost of a VR system can be prohibitive with both the initial purchase cost and ongoing maintenance fees.

\section{Simulation for New Surgical Technologies}

The acceleration of technological innovation in surgery continues with new techniques continually being introduced into 
surgical practice. Although innovation in surgery is crucial for the advancement of surgical practice, the introduction of new methods requires that both surgeons in training and those in practice safely and adequately learn new skills before operating on real patients. Simulation has the potential to provide a learning milieu to develop these new skills. Examples include single-incision laparoscopic surgery (SILS), natural orifice transluminal endoscopy surgery (NOTES), and robotic surgery.

\section{Single-Incision Laparoscopic Surgery}

Despite the rapid evolution of SILS in the general surgical realm, little is known about the safest and best way for surgeons to adopt this technique. The use of simulation to help surgeons acquire this skill set has been limited. Recently, Khandelwal et al published an approach to the adoption of SILS using simulation-based training. They developed a SILS simulator using a trainer box and surgical tasks based on the FLS program. In addition to box-training, their program involved performing a SILS cholecystectomy and appendectomy on a live nonsurvival porcine model. ${ }^{67}$ The authors describe a thoughtful curricular model for SILS training that could be adopted by other institutions. A randomized trial by Santos et al demonstrated that SILS-specific simulator training using FLS tasks resulted in improved SILS performance, ${ }^{68}$ providing evidence to support the use of simulation in the acquisition of SILS skills.

\section{Natural Orifice Transluminal Endoscopy Surgery}

NOTES surgery is based on both traditional laparoscopic and endoscopic principles and requires the collaborative acquisition of a unique skill set among endoscopists and minimally invasive surgeons. Since the first published NOTES report using a porcine model in $2004,{ }^{69}$ much of the subsequent development of this technology has occurred using porcine models and some cadaveric models. ${ }^{70-72}$ Up until recently, no specialized NOTES training platform existed. The ELITE platform (endoscopic-laparoscopic interdisciplinary training entity) has now been introduced for the ex vivo training of endoscopic, laparoscopic, and NOTES techniques such as cholecystectomy, appendectomy, and other gastrointestinal resections. ${ }^{73}$ The model consists of a life-size female adult with various transluminal accesses and synthetic intraabdominal organs. The ELITE is currently the only platform available for the acquisition of NOTES skills. Furthermore, evidence of construct validity has been demonstrated with expert endoscopists outperforming novices in terms of time to completion during a simulated ELITE procedure. ${ }^{74}$ Training on the ELITE platform has also been demonstrated to improve the performance of a NOTES cholecystectomy using a porcine model. ELITE-trained individuals complete the procedure in less time and have fewer complications and difficulties than their untrained counterparts. ${ }^{75}$

\section{Robotics}

Over the past few years, robotic surgery has become more widely adopted, especially in the area of urologic surgery. ${ }^{76}$ Platforms simulating the da Vinci robot have been developed and are now commercial available. The dV-Trainer by Mimic Technologies, Inc. (Seattle, WA) is a VR simulator that closely replicates the look and feel of the da Vinci console allowing trainees the opportunity to acquire robotic skills in a safe, low-stakes environment. Several studies have demonstrated evidence of face, content, and construct validity of the dVTrainer. ${ }^{77-79}$ Furthermore, training on the dV-Trainer has shown to result in improved robotic surgery performance. ${ }^{80}$ A recent randomized control trial by Korets et al demonstrated that training on the dV-Trainer, as well as training on the da Vinci console resulted in a significant improvement in robotic performance measured by several surgical parameters. $^{81}$

The RoSS (Robotic Surgery Simulator) by Simulated Surgical Systems, LLC, is another VR robotic system for the da Vinci robot (-Fig. 5). This platform replicates the da Vinci console and offers several surgical modules from basic skills to advanced surgical tasks. Full procedures including radical prostatectomy and hysterectomy are currently being developed and integrated into their system. ${ }^{82}$ The RoSS has demonstrated evidence of both face ${ }^{83}$ and content validity. ${ }^{84}$ Further validation of this system is currently underway.

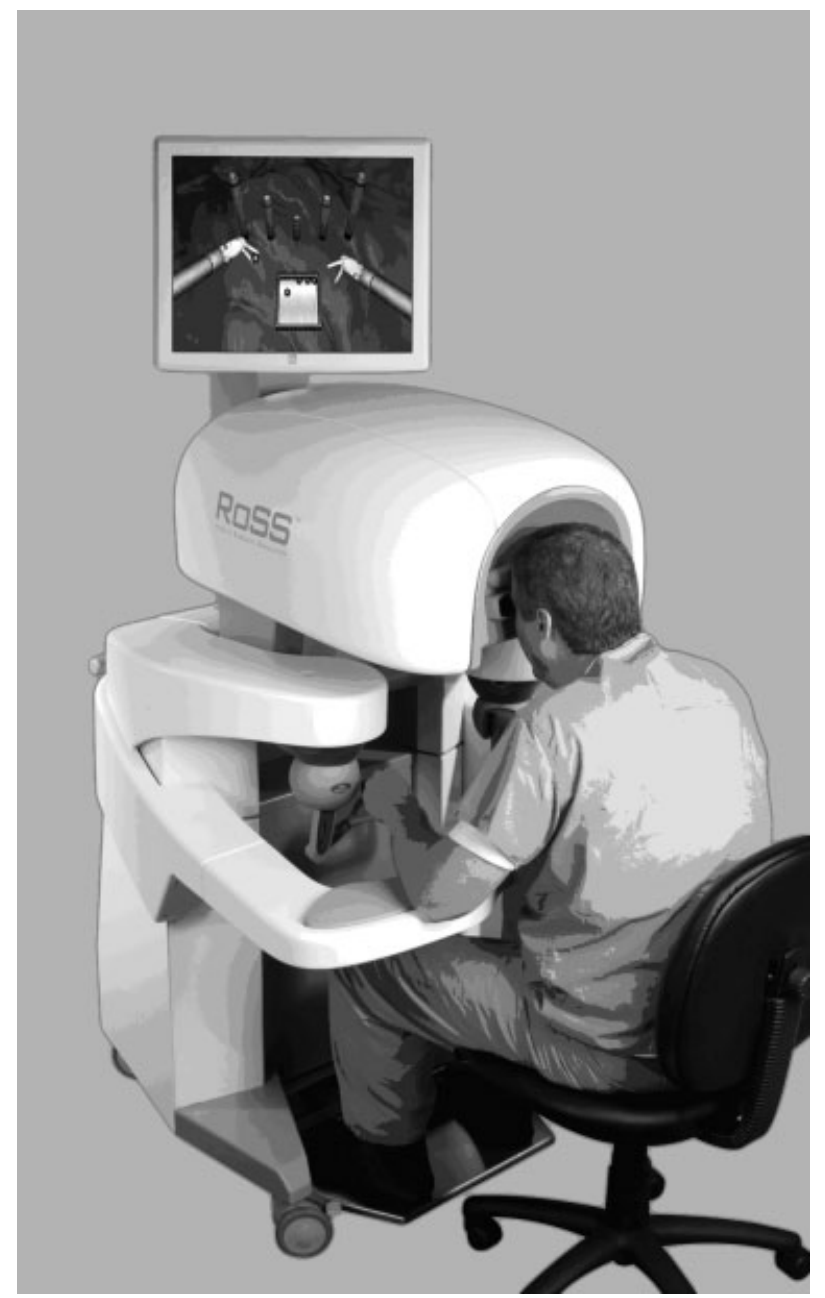

Figure 5 The RoSS ${ }^{\mathrm{TM}}$ robotic simulator platform. Image reproduced with permission from Simulated Surgical Systems, LLC (Williamsville, NY). 


\section{Simulation for Nontechnical Surgical Skills}

Most simulation in surgical education has focused on technical skills. However, nontechnical skills are essential to become a globally competent surgeon and simulation has been used for both teaching and assessing these skills. The Accreditation Council for Graduate Medical Education (ACGME) has defined the requisite components of medical competence. Two of these core competencies include professionalism and communication skills. $^{85}$

\section{Standardized Patients}

Standardized patients (SP) and standardized family members fall under the umbrella of simulation and have been incorporated into surgical training as a means to teach and evaluate nontechnical skills.

Yudkowsky et al developed an Objective Structured Clinical Exam (OSCE) to assess surgical residents' communication skills using SPs to simulate patient encounters. Residents were faced with several communication tasks such as delivering bad news, providing counseling and providing informed consent. The authors highlight the importance of this method of simulation as formative feedback where residents are given the opportunity to learn and reflect on their communication skills. ${ }^{86}$ The use of SPs in a plastic surgery OSCE has been shown to be an effective educational tool with the ability to provide comprehensive and meaningful feedback to trainees on professionalism and interpersonal communication. ${ }^{87}$

In addition to SPs, standardized family members have been used to simulate nontechnical skills scenarios. A randomized trial by Barrios et al investigated the communication skills of general surgery residents using simulation. A mock OR was used in which residents performed a laparoscopic cholecystectomy on a VR simulator. Residents were presented with either an iatrogenic common bile duct injury or the incidental finding of metastatic gallbladder cancer and required to deliver the news to a standardized family member. They found that residents were not well prepared to handle these difficult conversations and suggested simulation as a modality to improve communication skills in disclosing difficult information. ${ }^{88}$ Simulated family members were also used by Chipman et al who developed an OSCE for general surgery residents to practice and receive feedback on delivering bad news. Scenarios involved disclosing a terminal diagnosis and disclosing a surgical complication. ${ }^{89}$ Further study of this tool has demonstrated initial evidence of reliability; however, evidence of validity remains to be established. ${ }^{90}$

\section{Crisis Resource Management and Team Training}

Patient safety relies on the effective and efficient coordination among all health care professionals within the OR environment. Teamwork, communication, leadership, and judgment are all important factors in the success of an operation. The presence of multiple experts in the OR does not necessarily translate into expert team functioning. Furthermore, significant discrepancies exist in the perception of teamwork within the OR. A study by Makary et al found that surgeons were more likely to rate the teamwork of others as good, whereas nurses perceived teamwork as only mediocre. ${ }^{91}$ An increased awareness has arisen within the surgical community for the need to improve OR team training. Simulation is an ideal platform to meet this learning need.

Crew resource management, later referred to as crisis resource management (CRM) training was initially developed within the aviation industry after the realization that human factors, such as failed interpersonal communication, decision making, and leadership, were the leading cause of aviation error. $^{92}$ It was realized that flight crews needed to flatten the hierarchy, empower juniors to voice concerns, and teach senior members to respect these perspectives to improve the safety and efficiency of the team. ${ }^{93}$ Team training and CRM focuses on the development of skills such as dynamic decision making, interpersonal behavior, and team management during both ordinary and crisis situations. ${ }^{94}$ The principles of CRM have been applied to several domains of medicine including anesthesia, emergency medicine, critical care, and surgery. ${ }^{94}$ A recent systematic review looking at teamwork training by Zeltser and Nash found that anesthesia and surgery were the two most common specialties employing CRM training. ${ }^{95}$

Moorthy et al $^{96}$ developed and evaluated a surgical crisis scenario using simulation. Surgical trainees were faced with a bleeding crisis during the performance of a saphenofemoral ligation on a bench-top model in a simulated OR. Trainees were assessed both on technical and nontechnical skills including communication skills, situation awareness, team skills, leadership skills, and decision making. Although nontechnical skills did not demonstrate evidence of construct validity, the feasibility and realism of simulation-based team training was established.

The ultimate goal of team training is to improve patient care by improving team functioning. A recent study by Capella et al demonstrated that team training using simulation not only improves team performance, but also results in improved patient care. ${ }^{97}$ This pretraining/ posttraining study included surgical residents, faculty surgeons, and emergency department nurses. Team functioning was assessed in the trauma bay both before and after didactic and simulationbased team training. Team performance parameters including leadership, situation monitoring, mutual support, and communication improved significantly following training. Improved team functioning seemed to translate into better patient care by demonstrating a significantly improved time from arrival to intubation, computed tomography scan, and OR.

The potential of team training is only starting to be realized and its incorporation into surgical training is sure to grow as evidence of reliability and validity of the training and assessment of CRM are further established.

\section{Conclusions}

The ethical concern of learning a surgical craft on patients, financial constraints of the health care system, and work hour restrictions have required a change in the pedagogical approach to surgical training. The traditional model of training 
relied on the acquisition of surgical skills through an apprenticeship model. With advancement in our understanding of psychomotor skill learning and the development of validated simulation models, much of the early learning of surgical skills can occur in a laboratory setting. Simulation, in its various modalities, has dramatically changed the face of surgical education over the past decade by providing a platform for the acquisition of both technical and nontechnical surgical skills.

\section{References}

1 Satava RM, Gallagher AG, Pellegrini CA. Surgical competence and surgical proficiency: definitions, taxonomy, and metrics. J Am Coll Surg 2003;196(6):933-937

2 Cameron JL. William Stewart Halsted. Our surgical heritage. Ann Surg 1997;225(5):445-458

3 Kerr B, O'Leary JP. The training of the surgeon: Dr. Halsted's greatest legacy. Am Surg 1999;65(11):1101-1102

4 Reznick RK, MacRae H. Teaching surgical skills-changes in the wind. N Engl J Med 2006;355(25):2664-2669

5 Issenberg SB, McGaghie WC, Hart IR, et al. Simulation technology for health care professional skills training and assessment. JAMA 1999;282(9):861-866

6 Gaba DM. The future vision of simulation in health care. Qual Saf Health Care 2004;13(Suppl 1):i2-i10

7 Satava RM. Accomplishments and challenges of surgical simulation. Surg Endosc 2001;15(3):232-241

8 Ziv A, Wolpe PR, Small SD, Glick S. Simulation-based medical education: an ethical imperative. Acad Med:J Assoc Amer Med Coll 2003;78(8):783-788

9 Bruce PJ, Helmer SD, Osland JS, Ammar AD. Operative volume in the new era: a comparison of resident operative volume before and after implementation of 80-hour work week restrictions. J Surg Educ 2010;67(6):412-416

10 Rattner DW, Apelgren KN, Eubanks WS. The need for training opportunities in advanced laparoscopic surgery. Surg Endosc 2001;15(10):1066-1070

11 Palter VN, Orzech N, Aggarwal R, Okrainec A, Grantcharov TP. Resident perceptions of advanced laparoscopic skills training. Erratum in: Surg Endosc 2011;25(7):2408-2410

12 Bridges M, Diamond DL. The financial impact of teaching surgical residents in the operating room. Am J Surg 1999;177(1):28-32

13 Maran NJ, Glavin RJ. Low- to high-fidelity simulation-a continuum of medical education? Med Educ 2003;37(Suppl 1):22-28

14 Pikal MJ. Use of laboratory data in freeze drying process design: heat and mass transfer coefficients and the computer simulation of freeze drying. J Parenter Sci Technol 1985;39(3):115-139

15 Reznick R, Regehr G, MacRae H, Martin J, McCulloch W. Testing technical skill via an innovative "bench station" examination. Am J Surg 1997;173(3):226-230

16 Martin JA, Regehr G, Reznick R, et al. Objective structured assessment of technical skill (OSATS) for surgical residents. Br J Surg 1997;84(2):273-278

17 Faulkner H, Regehr G, Martin J, Reznick R. Validation of an objective structured assessment of technical skill for surgical residents. Acad Med 1996;71(12):1363-1365

18 Reznick RK, MacRae H. Teaching surgical skills-changes in the wind. N Engl J Med 2006;355(25):2664-2669

19 Regehr G, MacRae H, Reznick RK, Szalay D. Comparing the psychometric properties of checklists and global rating scales for assessing performance on an OSCE-format examination. Acad Med 1998;73(9):993-997

20 Siddiqui NY, Stepp KJ, Lasch SJ, Mangel JM, Wu JM. Objective structured assessment of technical skills for repair of fourth- degree perineal lacerations. Am J Obstet Gynecol 2008;199(6): 676, e1-e6

21 Lentz GM, Mandel LS, Lee D, Gardella C, Melville J, Goff BA. Testing surgical skills of obstetric and gynecologic residents in a bench laboratory setting: validity and reliability. Am J Obstet Gynecol 2001;184(7):1462-1468, discussion 1468-1470

22 Kingston A, Abbott J, Lenart M, Vancaillie T. Hysteroscopic training: the butternut pumpkin model. J Am Assoc Gynecol Laparosc 2004;11(2):256-261

23 Oyama IA, Aaronoff MC, Burlingame JM. Obstetric anal sphincter injury repair workshop for residents. Hawaii Med J 2009;68(6): 33-135

24 Lauscher JC, Ritz J-P, Stroux A, Buhr HJ, Gröne J. A new surgical trainer (BOPT) improves skill transfer for anastomotic techniques in gastrointestinal surgery into the operating room: a prospective randomized trial. World J Surg 2010;34(9):2017-2025

25 Gröne J, Lauscher JC, Buhr HJ, Ritz J-P. Face, content and construct validity of a new realistic trainer for conventional techniques in digestive surgery. Langenbecks Arch Surg 2010;395(5):581-588

26 Jensen AR, Wright AS, McIntyre LK, et al. Laboratory-based instruction for skin closure and bowel anastomosis for surgical residents. Arch Surg 2008;143(9):852-858, discussion 858-859

27 Brehmer M, Swartz R. Training on bench models improves dexterity in ureteroscopy. Eur Urol 2005;48(3):458-463, discussion 463

28 Anastakis DJ, Regehr G, Reznick RK, et al. Assessment of technical skills transfer from the bench training model to the human model. Am J Surg 1999;177(2):167-170

29 Grober ED, Hamstra SJ, Wanzel KR, et al. The educational impact of bench model fidelity on the acquisition of technical skill: the use of clinically relevant outcome measures. Ann Surg 2004;240(2): 374-381

30 Grober ED, Hamstra SJ, Wanzel KR, et al. Laboratory based training in urological microsurgery with bench model simulators: a randomized controlled trial evaluating the durability of technical skill. J Urol 2004;172(1):378-381

31 Matsumoto ED. Low-fidelity ureteroscopy models. J Endourol 2007;21(3):248-251

32 Matsumoto ED, Hamstra SJ, Radomski SB, Cusimano MD. The effect of bench model fidelity on endourological skills: a randomized controlled study. J Urol 2002;167(3):1243-1247

33 Sidhu RS, Park J, Brydges R, MacRae HM, Dubrowski A. Laboratorybased vascular anastomosis training: a randomized controlled trial evaluating the effects of bench model fidelity and level of training on skill acquisition. J Vasc Surg 2007;45(2):343-349

34 Datta V, Bann S, Beard J, Mandalia M, Darzi A. Comparison of bench test evaluations of surgical skill with live operating performance assessments. J Am Coll Surg 2004;199(4):603-606

35 Palter VN, Grantcharov T, Harvey A, Macrae HM. Ex vivo technical skills training transfers to the operating room and enhances cognitive learning: a randomized controlled trial. Ann Surg 2011;253(5):886-889

36 Dorman K, Satterthwaite L, Howard A, et al. Addressing the severe shortage of health care providers in Ethiopia: bench model teaching of technical skills. Med Educ 2009;43(7):621-627

37 Moore MJ, Bennett CL. The learning curve for laparoscopic cholecystectomy. The Southern Surgeons Club. Am J Surg 1995;170 (1):55-59

38 Rosser JC, Rosser LE, Savalgi RS. Skill acquisition and assessment for laparoscopic surgery. Arch Surg 1997;132(2):200-204

39 Khine M, Leung E, Morran C, Muthukumarasamy G. Homemade laparoscopic simulators for surgical trainees. Clin Teach 2011;8 (2):118-121

40 Kobayashi SA, Jamshidi R, O'Sullivan P, et al. Bringing the skills laboratory home: an affordable webcam-based personal trainer for developing laparoscopic skills. J Surg Educ 2011;68(2):105-109

41 Derossis AM, Fried GM, Abrahamowicz M, Sigman HH, Barkun JS, Meakins JL. Development of a model for training and evaluation of laparoscopic skills. Am J Surg 1998;175(6):482-487 
42 Dauster B, Steinberg AP, Vassiliou MC, et al. Validity of the MISTELS simulator for laparoscopy training in urology. J Endourol 2005;19 (5):541-545

43 Zheng B, Hur HC, Johnson S, Swanström LL. Validity of using Fundamentals of Laparoscopic Surgery (FLS) program to assess laparoscopic competence for gynecologists. Surg Endosc 2010;24 (1):152-160

44 Derossis AM, Bothwell J, Sigman HH, Fried GM. The effect of practice on performance in a laparoscopic simulator. Surg Endosc 1998;12(9):1117-1120

45 Fried GM, Derossis AM, Bothwell J, Sigman HH. Comparison of laparoscopic performance in vivo with performance measured in a laparoscopic simulator. Surg Endosc 1999;13(11):1077-1081, discussion 1082

46 McCluney AL, Vassiliou MC, Kaneva PA, et al. FLS simulator performance predicts intraoperative laparoscopic skill. Surg Endosc 2007;21(11):1991-1995

47 Sroka G, Feldman LS, Vassiliou MC, Kaneva PA, Fayez R, Fried GM. Fundamentals of laparoscopic surgery simulator training to proficiency improves laparoscopic performance in the operating rooma randomized controlled trial. Am J Surg 2010;199(1):115-120

48 Fried GM. FLS assessment of competency using simulated laparoscopic tasks. J Gastrointest Surg 2008;12(2):210-212

49 Fraser SA, Klassen DR, Feldman LS, Ghitulescu GA, Stanbridge D, Fried GM. Evaluating laparoscopic skills: setting the pass/fail score for the MISTELS system. Surg Endosc 2003;17(6):964-967

50 Taffinder N, Sutton C, Fishwick RJ, McManus IC, Darzi A. Validation of virtual reality to teach and assess psychomotor skills in laparoscopic surgery: results from randomised controlled studies using the MIST VR laparoscopic simulator. Stud Health Technol Inform 1998;50:124-130

51 Woodrum DT, Andreatta PB, Yellamanchilli RK, Feryus L, Gauger PG, Minter RM. Construct validity of the LapSim laparoscopic surgical simulator. Am J Surg 2006;191(1):28-32

52 van Dongen KW, Tournoij E, van der Zee DC, Schijven MP, Broeders IAMJ. Construct validity of the LapSim: can the LapSim virtual reality simulator distinguish between novices and experts? Surg Endosc 2007;21(8):1413-1417

53 Duffy AJ, Hogle NJ, McCarthy H, et al. Construct validity for the LAPSIM laparoscopic surgical simulator. Surg Endosc 2005;19(3): 401-405

54 Langelotz C, Kilian M, Paul C, Schwenk W. LapSim virtual reality laparoscopic simulator reflects clinical experience in German surgeons. Langenbecks Arch Surg 2005;390(6):534-537

55 Kundhal PS, Grantcharov TP. Psychomotor performance measured in a virtual environment correlates with technical skills in the operating room. Surg Endosc 2009;23(3):645-649

56 Hyltander A, Liljegren E, Rhodin PH, Lönroth H. The transfer of basic skills learned in a laparoscopic simulator to the operating room. Surg Endosc 2002;16(9):1324-1328

57 Torkington J, Smith SG, Rees BI, Darzi A. Skill transfer from virtual reality to a real laparoscopic task. Surg Endosc 2001;15(10): 1076-1079

58 Seymour NE, Gallagher AG, Roman SA, et al. Virtual reality training improves operating room performance: results of a randomized, double-blinded study. Ann Surg 2002;236(4):458-463, discussion 463-464

59 Grantcharov TP, Kristiansen VB, Bendix J, Bardram L, Rosenberg J, Funch-Jensen P. Randomized clinical trial of virtual reality simulation for laparoscopic skills training. Br J Surg 2004;91(2): $146-150$

60 Andreatta PB, Woodrum DT, Birkmeyer JD, et al. Laparoscopic skills are improved with LapMentor training: results of a randomized, double-blinded study. Ann Surg 2006;243(6):854-860, discussion 860-863

61 Delp SL, Loan JP, Hoy MG, Zajac FE, Topp EL, Rosen JM. An interactive graphics-based model of the lower extremity to study orthopaedic surgical procedures. IEEE Trans Biomed Eng 1990;37 (8):757-767

62 Larsen CR, Soerensen JL, Grantcharov TP, et al. Effect of virtual reality training on laparoscopic surgery: randomised controlled trial. BMJ 2009;338:b1802

63 Beyer L, Troyer JD, Mancini J, Bladou F, Berdah SV, Karsenty G. Impact of laparoscopy simulator training on the technical skills of future surgeons in the operating room: a prospective study. Am J Surg 2011;202(3):265-272

64 Sutherland LM, Middleton PF, Anthony A, et al. Surgical simulation: a systematic review. Ann Surg 2006;243(3):291-300

65 Gurusamy KS, Aggarwal R, Palanivelu L, Davidson BR. Virtual reality training for surgical trainees in laparoscopic surgery. Cochrane Database Syst Rev 2009;(1):CD006575

66 McDougall EM, Corica FA, Boker JR, et al. Construct validity testing of a laparoscopic surgical simulator. J Am Coll Surg 2006;202 (5):779-787

67 Khandelwal S, Wright AS, Figueredo E, Pellegrini CA, Oelschlager BK. Single-incision laparoscopy: training, techniques, and safe introduction to clinical practice. J Laparoendosc Adv Surg Tech A 2011;21(8):687-693

68 Santos BF, Reif TJ, Soper NJ, Hungness ES. Effect of training and instrument type on performance in single-incision laparoscopy: results of a randomized comparison using a surgical simulator. Surg Endosc 2011;25(12):3798-3804

69 Kalloo AN, Singh VK, Jagannath SB, et al. Flexible transgastric peritoneoscopy: a novel approach to diagnostic and therapeutic interventions in the peritoneal cavity. Gastrointest Endosc 2004;60(1):114-117

70 Moreira-Pinto J, Lima E, Correia-Pinto J, Rolanda C. Natural orifice transluminal endoscopy surgery: a review. World J Gastroenterol 2011;17(33):3795-3801

71 Hagen ME, Wagner OJ, Swain PC, et al. Transrectal natural orifice transluminal endoscopic surgery for umbilical hernia repair in a human cadaver (with video). Gastrointest Endosc 2009;69(6): e53-e54

72 Rieder E, Martinec DV, Dunst CM, Swanström LL. A novel technique for natural orifice endoscopic full-thickness colon wall resection: an experimental pilot study. J Am Coll Surg 2011;213 (3):422-429

73 Fiolka A, Gillen S, Meining A, Feussner H. ELITE-the ex vivo training unit for NOTES: development and validation. Minimally invasive therapy \& allied technologies. Minim Invasive Ther Allied Technol 2010;19(5):281-286

74 Gillen S, Wilhelm D, Meining A, et al. The "ELITE" model: construct validation of a new training system for natural orifice transluminal endoscopic surgery (NOTES). Endoscopy 2009;41(5): 395-399

75 Gillen S, Fiolka A, Kranzfelder M, et al. Training of a standardized natural orifice transluminal endoscopic surgery cholecystectomy using an ex vivo training unit. Endoscopy 2011;43(10): 876-881

76 Lee JY, Mucksavage P, Sundaram CP, McDougall EM. Best practices for robotic surgery training and credentialing. J Urol 2011;185 (4):1191-1197

77 Sethi AS, Peine WJ, Mohammadi Y, Sundaram CP. Validation of a novel virtual reality robotic simulator. J Endourol 2009;23(3): 503-508

78 Kenney PA, Wszolek MF, Gould JJ, Libertino JA, Moinzadeh A. Face, content, and construct validity of dV-trainer, a novel virtual reality simulator for robotic surgery. Urology 2009;73(6):1288-1292

79 Hung AJ, Zehnder P, Patil MB, et al. Face, content and construct validity of a novel robotic surgery simulator. J Urol 2011;186(3): 1019-1024

80 Lerner MA, Ayalew M, Peine WJ, Sundaram CP. Does training on a virtual reality robotic simulator improve performance on the da Vinci surgical system? J Endourol 2010;24(3):467-472 
81 Korets R, Mues AC, Graversen JA, et al. Validating the use of the Mimic dV-trainer for robotic surgery skill acquisition among urology residents. Urology 2011;78(6):1326-1330

82 Simulated Surgical Systems L. ROSS: Robotic surgery simulator. 2011. Available at: http://www.simulatedsurgicals.com/pdfs/RoSSBrochureSept2011.pdf. Accessed November 10, 2011

83 Seixas-Mikelus SA, Kesavadas T, Srimathveeravalli G, Chandrasekhar R, Wilding GE, Guru KA. Face validation of a novel robotic surgical simulator. Urology 2010;76(2):357-360

84 Seixas-Mikelus SA, Stegemann AP, Kesavadas T, et al. Content validation of a novel robotic surgical simulator. BJU Int 2011;107 (7):1130-1135

85 Stewart MG. Core Competencies. Accreditation Council for Graduate Medical Education website. 2001. Available at: http://www. acgme.org/acwebsite/RRC_280/280_corecomp.asp. Accessed September 20, 2011

86 Yudkowsky R, Alseidi A, Cintron J. Beyond fulfilling the core competencies: an objective structured clinical examination to assess communication and interpersonal skills in a surgical residency. Curr Surg 2004;61(5):499-503

87 Davis D, Lee G. The use of standardized patients in the plastic surgery residency curriculum: teaching core competencies with objective structured clinical examinations. Plast Reconstr Surg 2011;128(1):291-298

88 Barrios L, Tsuda S, Derevianko A, et al. Framing family conversation after early diagnosis of iatrogenic injury and incidental findings. Surg Endosc 2009;23(11):2535-2542
89 Chipman JG, Beilman GJ, Schmitz CC, Seatter SC. Development and pilot testing of an OSCE for difficult conversations in surgical intensive care. J Surg Educ 2007;64(2):79-87

90 Chipman JG, Webb TP, Shabahang M, et al. A multi-institutional study of the Family Conference Objective Structured Clinical Exam: a reliable assessment of professional communication. Am J Surg 2011;201(4):492-497

91 Makary MA, Sexton JB, Freischlag JA, et al. Operating room teamwork among physicians and nurses: teamwork in the eye of the beholder. J Am Coll Surg 2006;202(5):746-752

92 Helmreich RL, Merritt AC, Wilhelm JA. The evolution of Crew Resource Management training in commercial aviation. Int J Aviat Psychol 1999;9(1):19-32

93 Healy GB, Barker J, Madonna G. Error reduction through team leadership: applying aviation's CRM model in the OR. Bull Am Coll Surg 2006;91(2):10-15

94 Gaba DM. Crisis resource management and teamwork training in anaesthesia. Br J Anaesth 2010;105(1):3-6

95 Zeltser MV, Nash DB. Approaching the evidence basis for aviationderived teamwork training in medicine. Am J Med Qual 2010;25 (1):13-23

96 Moorthy K, Munz Y, Forrest D, et al. Surgical crisis management skills training and assessment: a simulation[corrected]-based approach to enhancing operating room performance. Ann Surg 2006;244(1):139-147

97 Capella J, Smith S, Philp A, et al. Teamwork training improves the clinical care of trauma patients. J Surg Educ 2010;67(6):439-443 